\title{
Peripheral blood inflammation indices are effective predictors of anastomotic leakage in elective esophageal surgery
}

\author{
Cai-Xia Wu ${ }^{1,2 \#}$, Ding-Yu Rao ${ }^{1,2 \#}$, Cheng-Peng Sang ${ }^{1,2 \#}$, Shen-Yu Zhu ${ }^{3}$, Liang Gu ${ }^{3}$, Yan-Yang Wu ${ }^{1}$, \\ Jian-Feng Wang ${ }^{4}$, Hua-Qiu Shi ${ }^{3}$, Xiang-Cai Wang ${ }^{4}$, Zhi-Xian Tang ${ }^{2,3}$ \\ ${ }^{1}$ First Clinical Medical College, The Gannan Medical University, Ganzhou, China; ${ }^{2}$ Ganzhou Key Lab of Brain Injury \& Brain Protection, Ganzhou, \\ China; ${ }^{3}$ Department of Thoracic Surgery, The First Affiliated Hospital of Gannan Medical University, Ganzhou, China; ${ }^{4}$ Department of Radiation \\ Oncology, The First Affiliated Hospital of Gannan Medical University, Ganzhou, China \\ Contributions: (I) Conception and design: CX Wu, XC Wang, ZX Tang; (II) Administrative support: None; (III) Provision of study materials or \\ patients: None; (IV) Collection and assembly of data: JF Wang, SY Zhu, YY Wu, DY Rao; (V) Data analysis and interpretation: JF Wang, SY Zhu, \\ CP Sang, CX Wu, L Gu; (VI) Manuscript writing: All authors; (VII) Final approval of manuscript: All authors. \\ \#These authors contributed equally to this work. \\ Correspondence to: Xiang-Cai Wang. Department of Radiation Oncology, The First Affiliated Hospital of Gannan Medical University, Ganzhou, \\ China. Email: wangxiangcai@csco.ac.cn; Zhi-Xian Tang. Department of Thoracic Surgery, The First Affiliated Hospital of Gannan Medical \\ University, Ganzhou, China. Email: tzhixian2020@gmu.edu.cn.
}

Background: This study investigated the predictive value of peripheral inflammatory indices, including neutrophil count, lymphocyte count, platelet count, neutrophil-to-lymphocyte ratio (NLR), and platelet-tolymphocyte ratio (PLR), in anastomotic leakage during elective esophageal surgery.

Methods: This retrospective study included all patients who underwent esophagectomy for esophageal squamous cell carcinoma from 2016 to 2020 in our institution. The peripheral blood inflammatory indices were obtained on preoperative days 1-7 (PRD 1-7), and postoperative days 1-3 (POD 1-3) and 4-7 (POD 4-7). Univariate, multivariate logistic, and receiver operating characteristic curve analyses were conducted to evaluate the diagnostic value of these peripheral blood inflammatory indices.

Results: A total of 198 patients were included in the study, and 25 (13\%) patients experienced anastomotic leakage. Multivariate analyses identified diet, neutrophil count, and PLR on POD 1-3, and NLR on POD 4-7 as independent factors associated with anastomotic leakage. Using the receiver operating characteristic curve, the variable with the best area under curve was a neutrophil cutoff count of 4.1 [0.737; 95\% CI: 0.639-0.835], with a sensitivity and specificity of $60.0 \%$ and $66.5 \%$, respectively. This was followed by an NLR cutoff value of 9.5 on POD 4-7 (0.628; 95\% CI: 0.505-0.752) and a cutoff PLR value of 220.1 on POD 1-3 (0.643; 95\% CI: 0.536-0.750). Diet showed a poor result on the receiver operating characteristic curve analysis.

Conclusions: Neutrophil count and PLR on POD 1-3 and NLR on POD 4-7 were shown to have predictive value for anastomotic leakage in elective esophageal surgery.

Keywords: Neutrophils; neutrophil-to-lymphocyte ratio (NLR); platelet-to-lymphocyte ratio (PLR); anastomotic leakage

Submitted Nov 16, 2021. Accepted for publication Dec 16, 2021.

doi: 10.21037/jgo-21-812

View this article at: https://dx.doi.org/10.21037/jgo-21-812

\section{Introduction}

According to the latest GLOBOCAN 2020 database, esophageal cancer ranks seventh in incidence and sixth in mortality in Asia, with a particularly high incidence in
China (1). Esophagectomy is widely acknowledged as the treatment that can best prolong the overall survival (OS) of patients with esophageal cancer. However, elective esophageal surgery is complex, involving important organs, 
such as the esophagus and the stomach, and has relatively high rates of complications. In fact, approximately $65 \%$ of patients report postoperative complications after esophagectomy (2), and the incidence of anastomotic leakage (AL) is $11.4 \%$ (3). Moreover, the Esophageal Complication Consensus Group has reported an increase in the incidence of AL from $11.7 \%$ to $13.1 \%$ (4).

Early fistulas occur within 3 days postsurgery and are related to the operation, mostly due to poor anastomosis, poor local blood supply, tension in the anastomosis, or errors in the application of the anastomosis. Early fistulas account for approximately $1-5 \%$ of anastomotic fistulas.

Medium-term fistulas occur 4-14 days after surgery and most commonly at about a week postoperatively. The causes are complex and include local suture infection of the anastomosis, necrosis of tissue cutting, untreated postoperative pleural effusion, incomplete lung expansion, poor tissue healing ability, severe and frequent coughing, chest and stomach dilatation, impaired gastric emptying, and pulling of the anastomosis by the stomach's own gravity. Medium-term fistulas account for approximately $75-85 \%$ of anastomotic fistulas.

Late fistulas present more than 14 days after surgery, mostly occur secondary to anastomotic fistulas, and are caused by local infections around the anastomosis or by small anastomotic fistula openings. This type of fistula is usually confirmed late clinically and accounts for about $10-20 \%$ of all fistulas.

Indeed, AL is one of the most severe complications after esophagectomy and can subsequently lead to peritonitis or mediastinitis, systemic inflammatory response syndrome (SIRS), and septic shock. It has been associated with high morbidity, prolonged hospital stay, and the high consumption of healthcare resources. In the longterm, AL can lead to a poor quality of life, increased cancer recurrence rates, and poor long-term survival (5). Therefore, early and timely diagnosis of esophageal $\mathrm{AL}$ is critical to avoiding potentially fatal complications and to reducing hospital stays and the clinical burden of patients with esophageal cancer. In general, when patients present with clinical symptoms, such as fever, chest pain, dyspnea, redness and swelling on the cervical anastomosis site, or production of secretions, then esophagography, computed tomography (CT) scans, or esophagoscopy are performed. Delayed diagnosis of AL can negatively influence prognosis. However, the timing of imaging remains controversial as the period of AL manifestation may vary considerably (6).

The reasons for the occurrence of anastomotic fistulas are complex and multifaceted, the most notable of which are closely related to anastomotic technique and the surgical operation. Other risk factors include frequent and violent postoperative coughing, which causes changes in the respiratory pressure being transmitted to the gastrointestinal tract, resulting in a massive shock wave of rapid pressure changes in the esophageal and gastric cavities. Swallowing large amounts of food and drink is also another risk factor which can result in pulling due to the stomach's own gravity. Both these factors can cause the fragile edematous anastomotic tissue to tear during the healing process and form anastomotic fistulas.

Surgeons today are witnessing rising infection-related complications following elective surgery, with in AL in particular being strongly associated with infection. AL can often lead to peritonitis or mediastinitis, with the latter being strongly associated with changes in circulating white blood cells and particularly with the presence of neutrophils and related lymphocytopenia (7). In general, a high neutrophil count predisposes an individual to inflammatory diseases. Conventionally available laboratory tests, such as blood cell counts, are easy to obtain, inexpensive, and acceptable to patients, and can therefore help to identify patients at high risk of complications. In particular, the neutrophil-to-lymphocyte ratio (NLR) can reflect systemic inflammatory responses, which is a reliable prognostic indicator in patients with advanced cancer (8). Studies have shown that the peripheral blood NLR before gastric cancer resection is predictive of AL (9). Furthermore, in patients undergoing elective colorectal surgery, the NLR on the fourth postoperative day has shown predictive value for AL (10). Other studies have indicated higher mean values and elevated NLR in the early postoperative period to be correlated with esophageal anastomosis in patients. Conversely, a low NLR has been reported to have high negative predictive value for AL (11). Although several studies have shown NLR to be an effective predictor of anastomotic leakage in elective esophageal surgery, these studies mainly measured the postoperative parameters, and thus could not provide insights into the preoperative inflammation indices with the potential to predict esophageal AL. Furthermore, the bulk of research is European in origin, and equivalent studies from Asia are lacking.

This current study aimed to evaluate and compare the predictive value of preoperative and postoperative inflammatory factors, such as neutrophil count, lymphocyte count, platelet count, NLR, platelet-to-lymphocyte ratio 


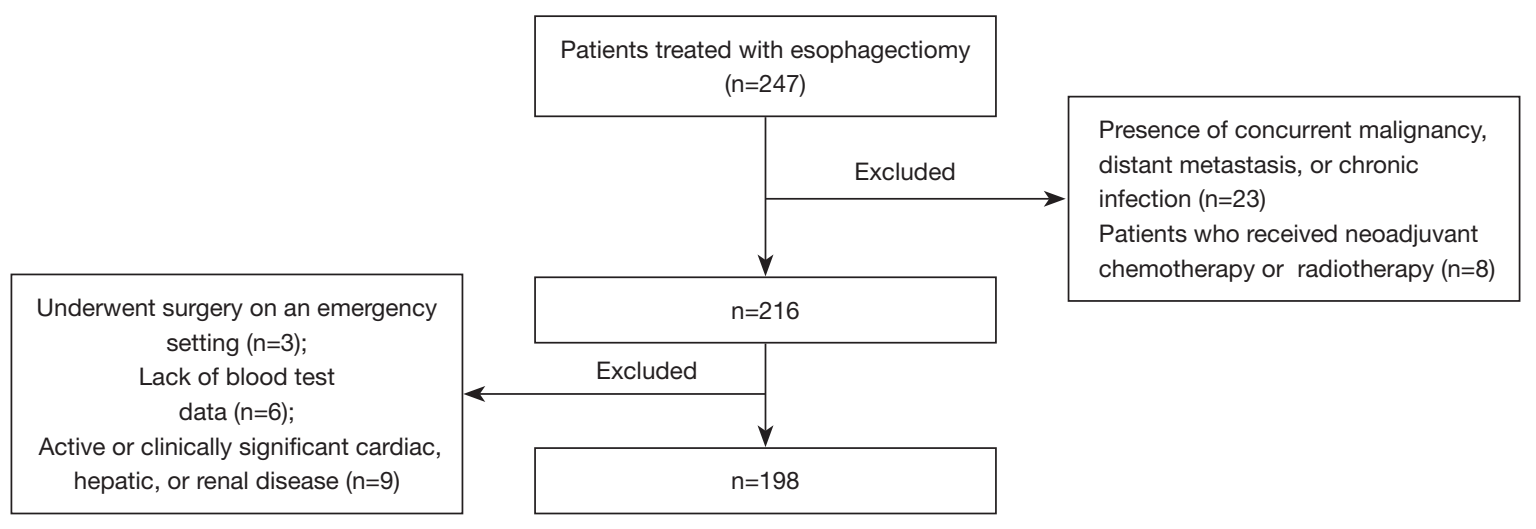

Figure 1 A flow diagram of the study protocol.

(PLR), and systemic immune-inflammation index (SII), in patients treated with curative resection for esophageal squamous cell carcinoma. In addition, the correlation of these peripheral blood inflammatory indices at the different AL times was examined.

We present the following article in accordance with the STARD reporting checklist (available at https://dx.doi. org/10.21037/jgo-21-812).

\section{Methods}

\section{Patient population}

This was a retrospective study on patients who underwent radical esophagectomy for esophageal cancer between 2016 and 2020 at the First Affiliated Hospital of Gannan Medical University. The demographic and clinicopathologic characteristics and blood test results were obtained from electronic or paper medical records. The inclusion criteria were as follows: (I) patients with histologically diagnosed esophageal squamous cell carcinoma, (II) patients who underwent elective esophageal surgical procedure, (III) complete availability of study variable data, (IV) complete availability of blood cell count data before and after surgery, and $(\mathrm{V})$ the absence of infections or inflammatory reactions 4 weeks before admission. The exclusion criteria were as follows: (I) age $<18$ or $>80$ years; (II) patients who received neoadjuvant chemotherapy or radiotherapy; (III) patients who underwent surgery under an emergency setting; (IV) incomplete blood test data; (V) active or clinically significant cardiac, hepatic, or renal disease; (VI) presence of concurrent malignancy, distant metastasis, or chronic infection; (VII) patients who were admitted to the intensive care unit after surgery; and (VIII) patients who experienced difficulty in expelling sputum after surgery. A total of 198 patients were eligible for this study (Figure 1). All patients provided written informed consent for surgery, and informed consent for this study was not required due its retrospective nature. All data were obtained from the electronic or paper medical records of The First Affiliated Hospital of Gannan Medical University and followed the data access policies. This study was conducted in accordance with the Declaration of Helsinki (as revised in 2013) and was exempt from ethical review by the ethics committee of Gannan Medical University.

\section{Variables}

The following variables were retrieved: age; gender; current smoking status; presence of lung disease, hypertension, or diabetes; diet; surgical approach, duration of operation, intraoperative blood loss, and blood transfusion; and tumor location and degree of differentiation. Data on peripheral blood inflammatory indices, such as neutrophil count, lymphocyte count, platelet count, NLR, PLR, and SII, were collated. The NLR was calculated by dividing the absolute neutrophil count by the absolute lymphocyte count. The PLR was calculated by dividing the absolute platelet count by the absolute lymphocyte count. The SII was defined as the platelet count multiplied by the neutrophil or lymphocyte counts. In addition, albumin and hemoglobin levels were considered in this study. These indices were taken at multiple time points: within 7 days preoperatively (PRD 1-7) and on postoperative days (POD) 1-3 and 4-7.

\section{Statistical analysis}

Statistical analysis was performed using the SPSS software, 
version 26 (IBM Corp., Armonk, NY, USA). Continuous data are reported as mean with standard deviation or as median with interquartile range. Categorical data are described as frequencies and percentages. Groups were compared using the chi-square test or Fisher's exact probability test for qualitative variables and the unpaired Student's $t$ test or Mann-Whitney rank-sum test for quantitative variables, as appropriate. The ShapiroWilcoxon test was used to assess normality of variable distribution. Correlations between variables were assessed by Friedman's correlation. Logistic regression analysis was used to investigate the associations of the peripheral inflammatory indices and the other risk factors with AL. Results were considered statistically significant with a $\mathrm{P}$ value $<0.05$ and a $95 \%$ CI.

The ability of the parameters to predict AL was examined using receiver operating characteristic (ROC) curve analysis, and the optimal cutoff with maximum sensitivity and specificity was selected. Sensitivity and specificity were reported using the optimal ROC curve values according to the Youden index. The results of the area under the curve (AUC) represented the global accuracy of the tests performed.

\section{Results}

\section{Patient characteristics}

The demographics, anthropometrics, and clinical data of the patients are summarized according to the presence or absence of AL (Table 1). A total of 198 patients, including $183(92.4 \%)$ males, with an average age of 61.3 years were enrolled in this study. There were $143(72.2 \%)$ current smokers. The most common type of diet before surgery was semiliquid (56.1\%). The comorbid diseases included hypertension in $29(14.6 \%)$ patients, diabetes in $6(3.0 \%)$ patients, and lung disease in $69(35.9 \%)$ patients. Open surgery was performed on 3 patients $(1.5 \%)$, with the most common esophagectomy procedure being the McKewon procedure (76.8\%), followed by Ivor Lewis surgery $(11.1 \%)$, and Sweet esophagectomy (10.6\%). Pretreatment clinical staging was performed based on a combination of CT images, endoscopic ultrasounds, and positron emission tomography (PET) scans. Tumor staging was performed according to the eighth edition of the American Joint Committee on Cancer (AJCC), and the majority of the patients were in stage III (55.9\%). In terms of tumor location, $65.5 \%$ of tumors were detected in the lower thoracic esophagus. The mean values for blood loss and operation time were $466.5 \mathrm{~mL}$ and 321.7 minutes, respectively.

\section{Anastomotic leakage}

A total of 25 of the 198 patients (13\%) included in the study were diagnosed with $\mathrm{AL}$ within 30 days. The mortality rate from AL was $12 \%(3 / 198)$. The incidence of cervical and thoracic AL was $40 \%(10 / 25)$ and $60 \%(15 / 25)$, respectively.

\section{Univariate and multivariate analysis of the factors associated with anastomotic leakage}

Univariate analysis was performed to determine the factors associated with AL (Table 2). As cytokines are not routine detection indicators, this study did not evaluate the relationship between cytokines and anastomotic leakage after esophageal surgery. Among the baseline data, only diet and operation time showed statistical significance. There were no significant differences in the median values of albumin, hemoglobin, platelets, or lymphocytes between patients with AL and those without AL. However, patients with AL had a significantly higher neutrophil count (3.81 vs. 5.53; $\mathrm{P}<0.000)$, NLR (2.67 vs. 3.83; $\mathrm{P}<0.000)$, PLR (175.58 vs. 188.07; $\mathrm{P}=0.058)$, and $\mathrm{SII}(663.94$ vs. $1,023.15 ; \mathrm{P}<0.000)$ compared to patients without AL. On POD 1-3, the mean lymphocyte count (1.00 vs. 0.75; $\mathrm{P}=0.035)$, NLR (13.99 vs. 17.76; $\mathrm{P}=0.013)$, PLR (295.46 vs. 374.23; $\mathrm{P}=0.021)$, and SII (3124.70 vs. 4039.07; $\mathrm{P}=0.007$ ) were consistently greater in patients who developed $\mathrm{AL}$ compared to patients who did not develop AL. On POD 4-7, only the neutrophil count (7.89 vs. 9.20; $\mathrm{P}=0.066)$ and the NLR (9.47 vs. 12.43; $\mathrm{P}=0.038$ ) were higher in patients with $\mathrm{AL}$ compared to those without AL. The Friedman test demonstrated that neutrophil count, NLR, and PLR on PRD 1-7, POD 1-3, and POD 4-7 were significantly different. Multivariate analysis (Table 3) revealed that the independent predictors of AL were diet [odds ratio (OR) 5.00, 95\% CI: 1.27-19.69; $\mathrm{P}=0.021$ ], neutrophil count (OR 1.80, 95\% CI: 1.36-2.39; $\mathrm{P}<0.001$ ), and PLR on POD 1-3 (OR 1.00, 95\% CI: $1.00-$ 1.01; $\mathrm{P}=0.022)$, and NLR on POD 4-7.

The ROC curve analysis (Table 3 and Figure 2) demonstrated that the best AUC was achieved for a preoperative neutrophil count cutoff value of 4.1 ( 0.737 ; 95\% CI: $0.639-0.835)$, with a sensitivity and specificity of $60.0 \%$ and $66.5 \%$, respectively, followed by a cutoff NLR value of 9.5 on POD $4-7$ (0.628; 95\% CI: $0.505-0.752)$ and 
Table 1 The main demographic, clinical, and surgical features of patients with and without anastomotic leakage

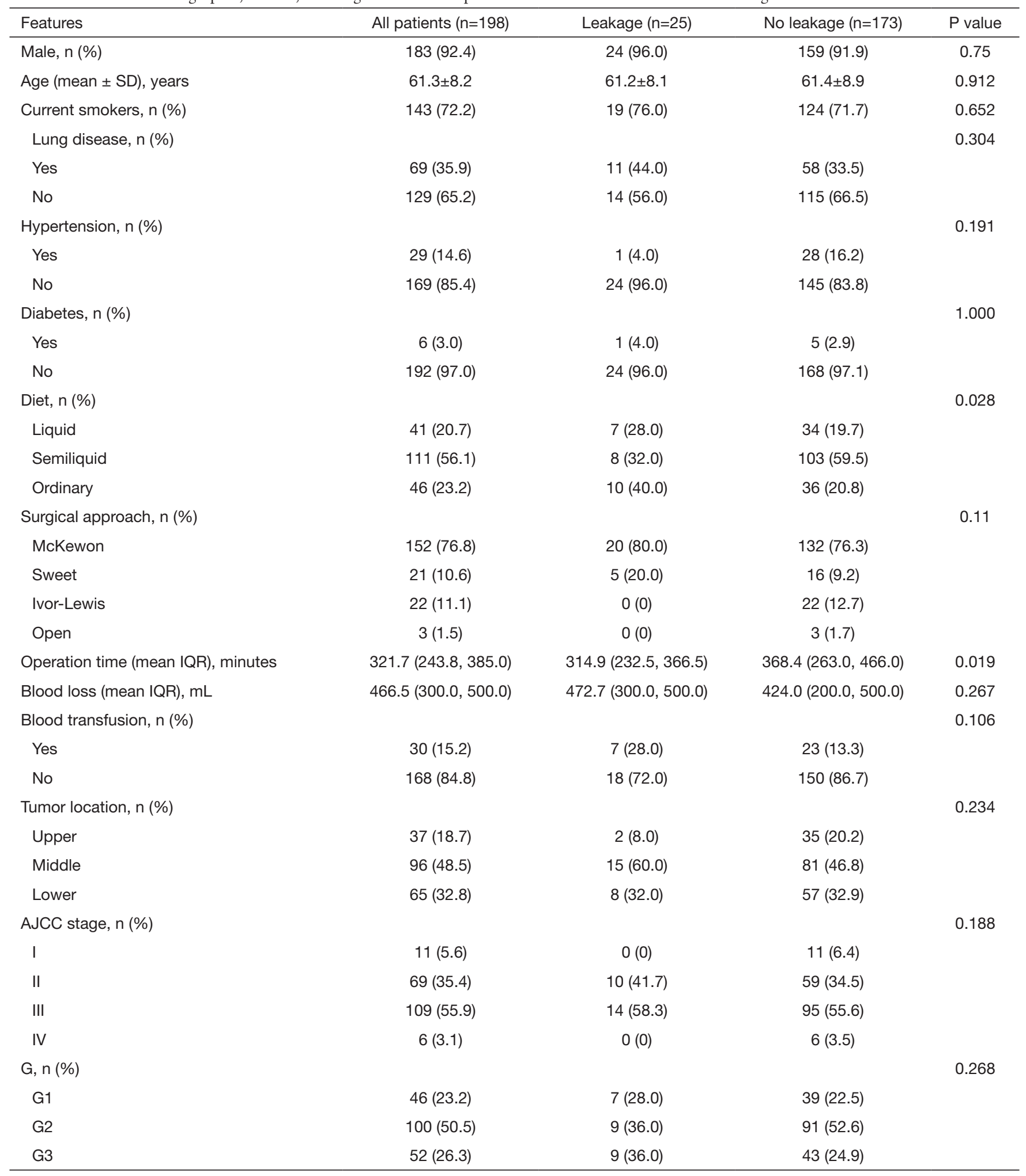

$\mathrm{SD}$, standard deviation; IQR, interquartile range; AJCC, American Joint Committee on Cancer. 
Table 2 A Comparison of the median values of the indexes studied preoperatively and at postoperative days 1-3 and days 4-7 in patients with and without anastomotic leakage

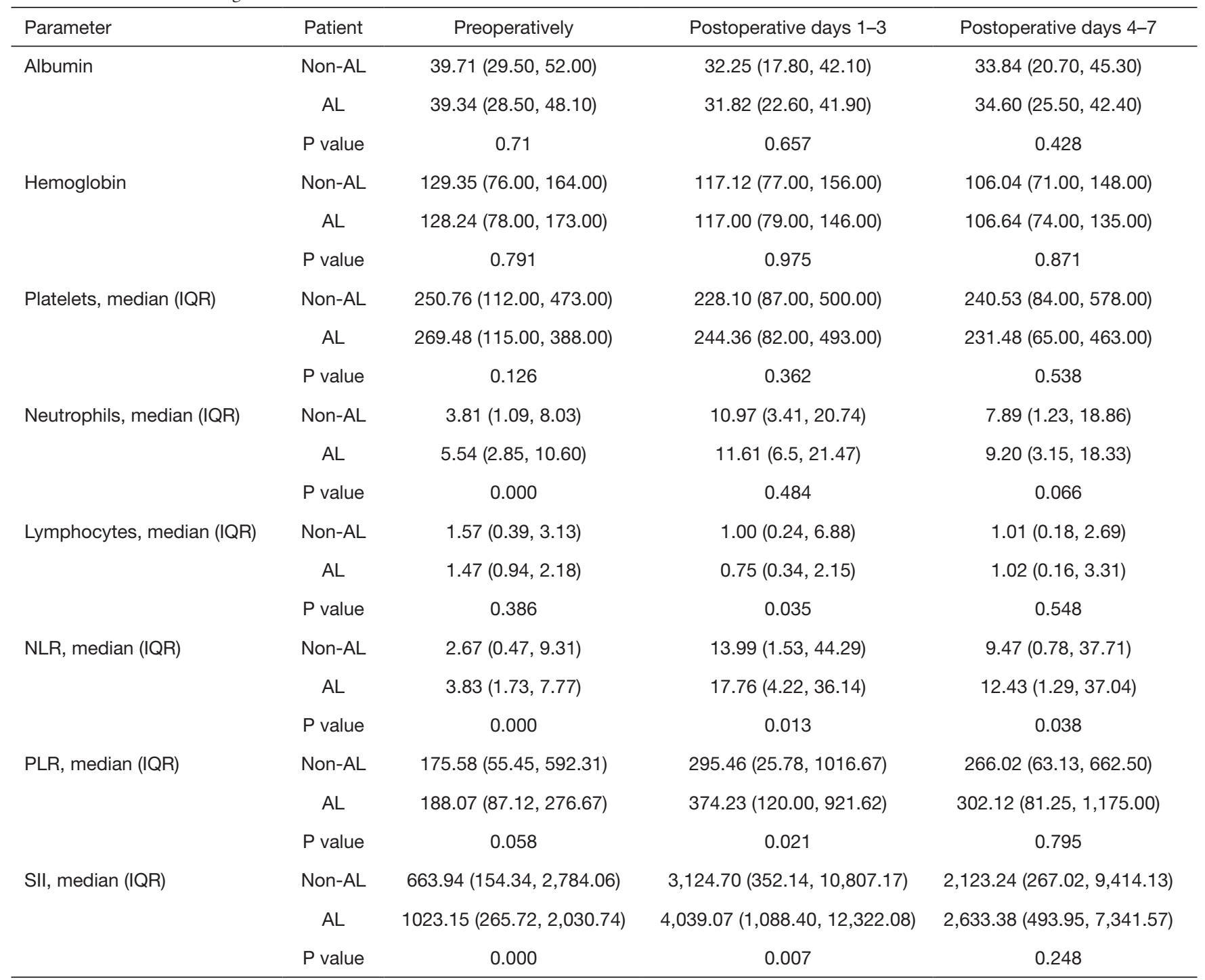

$\mathrm{AL}$, anastomotic leakage; IQR, interquartile range; NLR, neutrophil-to-lymphocyte ratio; PLR, platelet-to-lymphocyte ratio; SII, systemic immune-inflammation index.

Table 3 Receiver operating characteristic curves of the indexes under evaluation as predictive markers of anastomotic leakage

\begin{tabular}{lccccc}
\hline Marker & AUC & $95 \% \mathrm{Cl}$ & P value & Cutoff & Sensitivity \\
\hline Diet & 0.456 & $(0.317,0.595)$ & ns & & 0.72 \\
N & 0.737 & $(0.639,0.835)$ & 0.000 & 4.105 & 0.88 \\
D1-3 P-L & 0.643 & $(0.536,0.75)$ & 0.021 & 220.14 & 9.496 \\
D4-7 N-L & 0.628 & $(0.505,0.752)$ & 0.038 & 0.6 \\
\hline
\end{tabular}

AUC, area under the curve; ns, not significant; N, neutrophil; D1-3, postoperative days 1-3; D4-7, postoperative days 4-7; P-L, plateletto-lymphocyte; N-L, neutrophil-to-lymphocyte. 


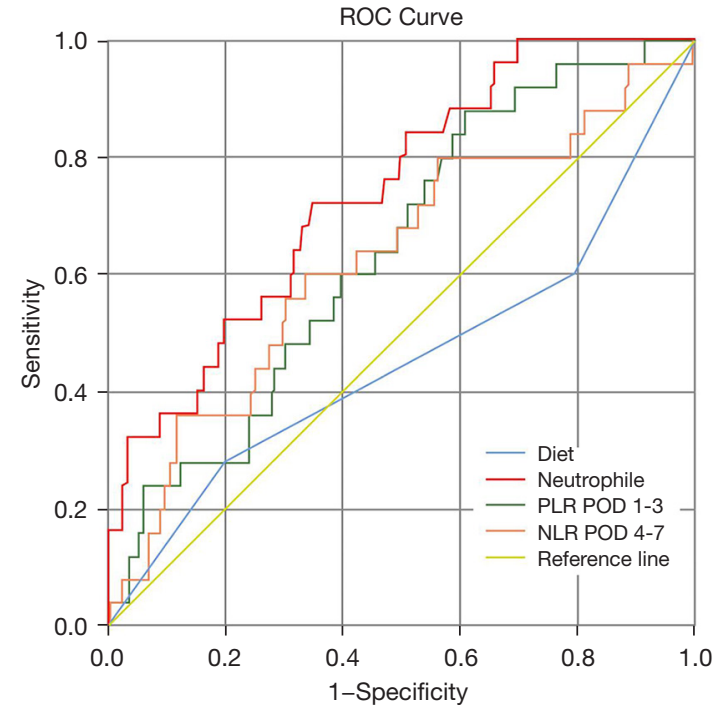

Figure 2 Receiver operating characteristic (ROC) curve.

a cutoff PLR value of 220.1 on POD $1-3(0.643,95 \%$ CI: 0.536-0.750). Diet showed a poor result on ROC analysis. Notably, PLR on POD1-3 had the best sensitivity (88.0\%) although its specificity was poor (39.3\%).

\section{Discussion}

Although findings concerning the mechanisms and predictive factors of AL have been equivocal, several studies have attempted to clarify these by analyzing the clinical data of patients with esophageal cancer. The Society of Thoracic Surgeons General Thoracic Database reported heart failure, hypertension, renal insufficiency, and type of procedure as risk factors for the development of AL (12). The incidence of cervical AL was reported to be significantly higher than that of thoracic AL (13), and anastomotic technique and position, thoracic approach, and the surgical method may affect the development of postoperative AL (14-16).

Several recent studies have shown that inflammation associated with malignant tumors can promote local development, tumor progression, and hematologic dissemination $(17,18)$. When inflammation and infections occur, neutrophils are the first line of defense and migrate to the sites of inflammation to protect against invading pathogens. A rapid influx of blood neutrophils characterizes the onset and is the hallmark of the inflammatory response. In fact, a high neutrophil count has been reported to promote the development and metastasis of cancer $(19,20)$. Moreover, in one study, the lymphocytes involved in the immune status decreased as the inflammatory disease progressed, but this decrease was relatively delayed and may not reflect the disease progression well (21). Compared with neutrophils or lymphocytes alone, NLR has a better predictive value in patient survival (22). Indeed, elevated peripheral blood NLR has recently become an independent prognostic indicator of poor overall survival in patients with cancer (23-25). A large number of studies have reported that AL may be associated with serum inflammatory indices, such as neutrophils, C-reactive protein (CRP), and NLR.

In a study by Radulescu et al. examining 204 gastric cancer patients who underwent radical gastric surgery, the preoperative NLR obtained upon initial presentation was significantly associated with AL, and an NLR > 3.54 was found to be an independent predictor of $\mathrm{AL}$ (OR 17.62; $\mathrm{P}<0.01)$ and complications leading to death $(\mathrm{P}<0.025)(9)$. Miyakita et al. demonstrated that a preoperative NLR cutoff of 2.21 was an independent predictor of AL in patients with rectal cancer (OR 8.24; $\mathrm{P}=0.0089$ ), with a sensitivity and specificity of $83 \%$ and $47 \%$, respectively (26). This cutoff was much lower than the median values of preoperative NLR reported in many other studies. Our current study showed similar results and did not identify a significant difference in the preoperative NLR between the 2 groups of patients. However, a neutrophil count of 4.1 before elective esophageal surgery had the best AUC in predicting AL, with a sensitivity of $60.0 \%$ and a specificity of $66.5 \%$. The neutrophil counts at the 3 different time points were significantly different. These results suggested that in patients with a neutrophil count $\geq 4.1$, measures should be taken to address the cause and prevent the development of AL.

Most studies stressed the fact that NLR on POD 4 reached statistical significance. Walker at el. reported that NLR is a useful predictor of AL with an AUC of 0.78 on POD (27). Paliogiannis and colleagues examined 1432 consecutive patients who underwent colorectal surgery and identified that a NLR cutoff value of 7.1 on POD 4 showed the best AUC in predicting AL (0.744; 95\% CI: 0.719 $0.768)(10)$. In another study of esophageal AL, mean NLR values demonstrated an increasing trend and were relatively high in the early postoperative period; conversely, a low NLR was associated with a high negative predictive value for AL (11).

This current study excluded patients who were on neoadjuvant chemotherapy or radiotherapy, which had been reported to have an impact on the development of AL (28). In order to control for confounding factors and improve data accuracy, patients who presented with concurrent 
malignancies, distant metastasis, or chronic infections were excluded. In this study population, esophagectomy was performed by experienced surgeons, and the most common type of surgery was the McKewon procedure. Univariate analysis revealed that operation time was a significant factor associated with AL, but this could not be confirmed with multivariate analysis. Moreover, surgical approach, volume of blood loss, and blood transfusion were not associated with AL. Rather, the peripheral blood inflammatory indices, including neutrophil count and PLR on POD 1-3, and the NLR on POD 4-7, were significant predictors of AL development. The significance of the lymphocyte count on POD 1-3 may be attributed to a delayed physiological response.

The diagnosis of esophageal AL mainly depends on clinical symptoms and imaging examination, which can contribute to poor clinical outcomes in patients. Consequently, the early prediction of esophageal AL is critical. Most studies show that esophageal AL is related to the increase of NLR on the fourth day after operation $(10,11)$. Our study further confirmed that serum inflammatory indicators, excluding postoperative NLR, are related to esophageal AL and demonstrated that preoperative neutrophil and POD1-3 PLR are associated with esophageal AL. This further proves that peripheral blood inflammatory indexes associate with esophageal AL. Using these simple and low-cost parameters for predicting esophageal AL, thoracic surgeons can focus on patients with high preoperative and postoperative inflammatory indicators, and avoid the occurrence of $\mathrm{Al}$ through the active, early, and appropriate preventative use of antibiotics. This would reduce the medical burden of patients undergoing radical esophagectomy and improve the quality of life and prognosis. Based on these results, the simple, inexpensive, reproducible, and widely available parameters of neutrophil count, lymphocyte count, NLR, and PLR can be used as predictive makers of AL after elective esophageal surgery in the clinical setting.

There were several limitations to this study. First, this investigation was performed in a single-centered setting and the sample size was small. In addition, the combination of early postoperative imaging data may be more efficient in diagnosing AL. Future multicenter studies using a large number of patients should be conducted to verify these results.

\section{Conclusions}

Neutrophil count and PLR on POD $1-3$ and NLR on
POD 4-7 are independent predictive factors of AL. Patients with relatively high neutrophil count and PLR on POD 1-3 and NLR on POD 4-7 may be more likely to develop AL. Therefore, peripheral blood inflammatory indices can serve as predictors of $\mathrm{AL}$ after elective esophageal surgery.

\section{Acknowledgments}

Funding: This study was funded by National Natural Science Funds of China (Grant No. 81860337, 81960326, 82060384), Natural Science Funds of Jiangxi Province (Grant No. 20202ACBL206014, 20192BAB205009, 2020BABL206116), Science \&Technology Programn of Jiangxi Health Commission (Grant No. 20201080, 202130660), National Health Commission Science \&Technology Development Research Center (Grant No. 2019ZH-07E-003), General projects of Jiangxi Traditional Chinese Medicine Science and Technology (Grant No. 2020B0214).

\section{Footnote}

Reporting Checklist: The authors have completed the STARD reporting checklist. Available at https://dx.doi. org/10.21037/jgo-21-812

Data Sharing Statement: Available at https://dx.doi. org/10.21037/jgo-21-812

Conflicts of Interest: All authors have completed the ICMJE uniform disclosure form (available at https://dx.doi. org/10.21037/jgo-21-812). The authors have no conflicts of interest to declare.

Ethical Statement: The authors are accountable for all aspects of the work in ensuring that questions related to the accuracy or integrity of any part of the work are appropriately investigated and resolved. All data were obtained from the electronic or paper medical records of The First Affiliated Hospital of Gannan Medical University and followed the data access policies. All patients provided written informed consent for surgery, and informed consent for this study was not required due its retrospective nature. This study was conducted in accordance with the Declaration of Helsinki (as revised in 2013) and was exempt from ethical review by the ethics committee of Gannan Medical University.

Open Access Statement: This is an Open Access article 
distributed in accordance with the Creative Commons Attribution-NonCommercial-NoDerivs 4.0 International License (CC BY-NC-ND 4.0), which permits the noncommercial replication and distribution of the article with the strict proviso that no changes or edits are made and the original work is properly cited (including links to both the formal publication through the relevant DOI and the license). See: https://creativecommons.org/licenses/by-nc-nd/4.0/.

\section{References}

1. Sung H, Ferlay J, Siegel RL, et al. Global Cancer Statistics 2020: GLOBOCAN Estimates of Incidence and Mortality Worldwide for 36 Cancers in 185 Countries. CA Cancer J Clin 2021;71:209-49.

2. van der Werf LR, Busweiler LAD, van Sandick JW, et al. Reporting National Outcomes After Esophagectomy and Gastrectomy According to the Esophageal Complications Consensus Group (ECCG). Ann Surg 2020;271:1095-101.

3. Low DE, Kuppusamy MK, Alderson D, et al. Benchmarking Complications Associated with Esophagectomy. Ann Surg 2019;269:291-8.

4. Kuppusamy MK, Low DE; International Esodata Study Group (IESG). Evaluation of International Contemporary Operative Outcomes and Management Trends Associated With Esophagectomy: A 4-Year Study of >6000 Patients Using ECCG Definitions and the Online Esodata Database. Ann Surg 2020. [Epub ahead of print]. doi: 10.1097/SLA.0000000000004309.

5. Hua X, Qian R, Shi K, et al. Effectiveness and safety of bovine pericardium patch repair for cervical anastomotic leakage after oesophagectomy for cancer. J Thorac Dis 2019;11:3808-13.

6. Messager M, Warlaumont M, Renaud F, et al. Recent improvements in the management of esophageal anastomotic leak after surgery for cancer. Eur J Surg Oncol 2017;43:258-69.

7. Ackland GL, Minto G, Clark M, et al. Autonomic regulation of systemic inflammation in humans: A multicenter, blinded observational cohort study. Brain Behav Immun 2018;67:47-53.

8. Dolan RD, Lim J, McSorley ST, et al. The role of the systemic inflammatory response in predicting outcomes in patients with operable cancer: Systematic review and metaanalysis. Sci Rep 2017;7:16717.

9. Radulescu D, Baleanu VD, Padureanu V, et al. Neutrophil/ Lymphocyte Ratio as Predictor of Anastomotic Leak after Gastric Cancer Surgery. Diagnostics (Basel) 2020;10:799.
10. Paliogiannis P, Deidda S, Maslyankov S, et al. Blood cell count indexes as predictors of anastomotic leakage in elective colorectal surgery: a multicenter study on 1432 patients. World J Surg Oncol 2020;18:89.

11. Al Lawati Y, Alkaaki A, Luis Ramírez García Luna J, et al. The Predictive Value of Inflammatory Biomarkers in Esophageal Anastomotic Leaks. Ann Thorac Surg 2021;112:1790-6.

12. Kassis ES, Kosinski AS, Ross P Jr, et al. Predictors of anastomotic leak after esophagectomy: an analysis of the society of thoracic surgeons general thoracic database. Ann Thorac Surg 2013;96:1919-26.

13. Gooszen JAH, Goense L, Gisbertz SS, et al. Intrathoracic versus cervical anastomosis and predictors of anastomotic leakage after oesophagectomy for cancer. Br J Surg 2018;105:552-60.

14. van Workum F, Slaman AE, van Berge Henegouwen MI, et al. Propensity Score-Matched Analysis Comparing Minimally Invasive Ivor Lewis Versus Minimally Invasive Mckeown Esophagectomy. Ann Surg 2020;271:128-33.

15. Schröder W, Raptis DA, Schmidt HM, et al. Anastomotic Techniques and Associated Morbidity in Total Minimally Invasive Transthoracic Esophagectomy: Results From the EsoBenchmark Database. Ann Surg 2019;270:820-6.

16. Grimminger PP, Staubitz JI, Perez D, et al. Multicenter Experience in Robot-Assisted Minimally Invasive Esophagectomy - a Comparison of Hybrid and Totally Robot-Assisted Techniques. J Gastrointest Surg 2021;25:2463-9.

17. Bondar T, Medzhitov R. The origins of tumor-promoting inflammation. Cancer Cell 2013;24:143-4.

18. Blaisdell A, Crequer A, Columbus D, et al. Neutrophils Oppose Uterine Epithelial Carcinogenesis via Debridement of Hypoxic Tumor Cells. Cancer Cell 2015;28:785-99.

19. Zhang J, Zhang Y, Yv X, et al. Prognostic value of combined preoperative prognostic nutritional index and neutrophil to lymphocyte ratio in esophageal squamous cell carcinoma. Transl Cancer Res 2020;9:5117-27.

20. Mollinedo F. Neutrophil Degranulation, Plasticity, and Cancer Metastasis. Trends Immunol 2019;40:228-42.

21. Vidal AC, Howard LE, de Hoedt A, et al. Neutrophil, lymphocyte and platelet counts, and risk of prostate cancer outcomes in white and black men: results from the SEARCH database. Cancer Causes Control 2018;29:581-8.

22. Kumarasamy C, Sabarimurugan S, Madurantakam RM, et al. Prognostic significance of blood inflammatory 
biomarkers NLR, PLR, and LMR in cancer-A protocol for systematic review and meta-analysis. Medicine (Baltimore) 2019;98:e14834.

23. Kim JH, Son NH, Lee JS, et al. Time-Sequencing of the Neutrophil-to-Lymphocyte Ratio to Predict Prognosis of Triple-Negative Breast Cancer. Cancers (Basel) 2021;13:3472.

24. Arvanitakis K, Mitroulis I, Germanidis G. TumorAssociated Neutrophils in Hepatocellular Carcinoma Pathogenesis, Prognosis, and Therapy. Cancers (Basel) 2021;13:2899.

25. Zheng Z, Lin D, Chen Q, et al. Prognostic Value of Combined Detection of Preoperative Albumin-toFibrinogen Ratio and Neutrophil-to-Lymphocyte Ratio in
Operable Esophageal Squamous Cell Carcinoma Patients without Neoadjuvant Therapy. Cancer Manag Res 2021;13:2359-70.

26. Miyakita H, Sadahiro S, Saito G, et al. Risk scores as useful predictors of perioperative complications in patients with rectal cancer who received radical surgery. Int J Clin Oncol 2017;22:324-31.

27. Walker PA, Kunjuraman B, Bartolo DCC. Neutrophil-tolymphocyte ratio predicts anastomotic dehiscence. ANZ J Surg 2018. [Epub ahead of print]. doi: 10.1111/ans.14369.

28. Goense L, van Rossum PSN, Ruurda JP, et al. Radiation to the Gastric Fundus Increases the Risk of Anastomotic Leakage After Esophagectomy. Ann Thorac Surg 2016;102:1798-804.

Cite this article as: Wu CX, Rao DY, Sang CP, Zhu SY, Gu L, Wu YY, Wang JF, Shi HQ, Wang XC, Tang ZX. Peripheral blood inflammation indices are effective predictors of anastomotic leakage in elective esophageal surgery. J Gastrointest Oncol 2021;12(6):2675-2684. doi: 10.21037/jgo-21812 